\title{
NONDESTRUCTIVE DETECTION OF THE INTERNAL QUALITY OF APPLE USING X-RAY AND MACHINE VISION
}

\author{
Fuzeng Yang ${ }^{1,2, *}$, Liangliang Yang ${ }^{1}$, Qing Yang ${ }^{1}$, Likui Kang ${ }^{1}$ \\ ${ }^{1}$ College of Mechanical and Electronic Engineering, Northwest Agriculture and Forestry \\ University, Yangling, China, 712100 \\ ${ }^{2}$ Department of Computer Science and Technology, Northwestern Polytechnical University, \\ Xi'an, China, 710072 \\ * Corresponding author, Address: College of Mechanical and Electronic Engineering, \\ Northwest Agriculture and Forestry University, Yangling, 712100, P. R. China, Tel: +86- \\ 29-87092913, +86-13772025795, Email:yfz0701@163.com
}

Abstract: The internal quality of apple is impossible to be detected by eyes in the procedure of sorting, which could reduce the apple's quality reaching market. This paper illustrates an instrument using X-ray and machine vision. The following steps were introduced to process the X-ray image in order to determine the mould core apple. Firstly, lifting wavelet transform was used to get a low frequency image and three high frequency images. Secondly, we enhanced the low frequency image through image's histogram equalization. Then, the edge of each apple's image was detected using canny operator. Finally, a threshold was set to clarify mould core and normal apple according to the different length of the apple core's diameter. The experimental results show that this method could on-line detect the mould core apple with less time consuming, less than 0.03 seconds per apple, and the accuracy could reach $92 \%$.

Keywords: X-ray; machine vision; detection; apple; internal quality.

\section{INTRODUCTION}

Mould core of apple could be aroused by Trichothecium roseum and other factors (Zhang et al., 2008). The fumaric acid in apple juice increases

Please use the following format when citing this chapter:

Yang, F., Yang, L., Yang, Q. and Kang, L., 2009, in IFIP International Federation for Information Processing, Volume 295, Computer and Computing Technologies in Agriculture II, Volume 3, eds. D. Li, Z. Chunjiang, (Boston: Springer), pp. 1699-1706. 
dramatically from 0.8 to 2.6 due to the mould core (Yi et al., 2001). Therefore, it is necessary to detect the internal quality to guarantee the quality of apple reaching market.

In the past few years, there are many methods which have been proposed to detect internal quality of fruits and vegetables. Multi spectroscopy has been investigated for detecting the brown heart of pear (Zerbini et al., 2002; Han et al., 2006). Near-Infrared Spectroscopy (NIR) has been used for predicting sugar content of Apple (Ying et al., 2006). Ultraviolet (UV) fluorescence has been reported for detecting freeze damage of citrus (Slaughter et al., 2008). Researches has been done at using Magnetic resonance imaging (MRI) (Thybo et al., 2004), laser technique methods to measure fruit tissue texture and internal disorders (Muramatsu et al., 1999). X-ray technology has also been adopted for detecting the agricultural quarantine materials (Toyofuku et al., 2007), seed weevil-infested mango fruits (Thomas et al., 1995) and the edible ratio and sugar content of rambuta (Zhang et al., 2005). However, there is little report on the detection of mould core apple using X-ray. It is necessary to do research on detecting the apple's internal quality using low-cost X-ray instrument.

Furthermore, digital image processing algorithm is vital in detecting and extracting the image features, such as brown heart in pear (Zerbini et al., 2002; Han et al., 2006), stem-end injury in citrus (Blasco et al., 2007) and edible ratio and sugar content of rambuta (Zhang et al., 2005). The algorithms were focused on spatial domain in former researches. In recent two decades, Wavelet Transform (WT) from Multi Resolution Analysis (MRA) has been successfully used in image processing, which can detect the signal catastrophe point in spatial and frequency domain. The WT is known as the mathematic microscope (Daubechies, 1992; Yang, 2007). Ying et al. (2006) has used the WT de-noising NIR image of apple; the results showed WT can improve the de-noising effort. Yang et al. $(2005,2007)$ has done many researches in agricultural image de-nosing and enhancement, the results showed the WT is an effective algorithm in agricultural image processing. In 1990's Sweldens et al. provided the Second Generation Wavelet Transform - Lifting Wavelet Transform (LWT), which provides a quick and less memory needed algorithm in frequency domain (Sweldens, 1995, 1996). However, there are not any researches on agricultural image processing using LWT.

The objectives of this study are to:

(1) Construct a real time detection system to detect the internal quality of apple using X-ray.

(2) Determine a suitable image processing algorithm to extract image's features using lifting scheme wavelet in frequency domain, which could efficiently detect the mould core apple. 
(3) Determine a processing method, using the (2) algorithm, can efficiently classify the apples with or without mould core, and determine its accuracy.

\section{MATERIALS AND METHODS}

\subsection{Apple samples}

One hundred apple samples were harvested in Mid-September 2007 from an orchard in Yangling, Shaanxi province of China and stored without any special instruments. The apples were transported to the laboratory in March 2008 , and then stored in the room condition.

\subsection{Instrumentation}

The real-time internal quality detection system consists of two parts. The first part is an X-ray radiator (BJI-1U, Bo Jin Electronic Instrument, Inc., Shanghai, China) and a machine vision system, which includes a black and white (BW) camera (ECB-1793, Hong Tian Zhi Electronic, Inc., Shenzhen, China) and an image grabber (BS-602A, Bao Shi, Inc., Taiwan, China). The other is an X-ray image processing workstation (Itellistation Z Pro MT: 9228, IBM, New York) with the image processing program programmed by ourselves using the Matlab (MathWorks, Inc., Natick, Mass).

\subsection{Detecting the mould core of apple}

The prime purpose of the system is to determine whether the apple is a mould core apple. The success of the detection depends mostly on the correct image feature extraction. The following sections will focus on how to extract image features.

\subsubsection{X-ray image processing algorithm}

We proposed an effective algorithm that is used to detect the mould core apple in frequency domains inspired by the LWT. 


\subsubsection{Lifting scheme wavelet}

A canonical case of lifting consists of three stages: split, predict, and update (Sweldens, 1995, 1996). It is simply described as follows.

Firstly, the signal $\lambda_{0}$ is divided in to two parts $\lambda_{-1}$ and $\gamma_{-1}$.

Secondly, we use the $\lambda_{-1}$ subset to predict the $\gamma_{-1}$ subset based on the correlation present in the original data. If a prediction operator $\mathrm{P}$ can be found,

$$
g_{-1}=P\left(l_{-1}\right) .
$$

If the prediction is reasonable, the difference will contain much less information than the original $\gamma_{-1}$ set. We can denote this abstract difference operator as

$$
g_{-1}=g_{-1}-P\left(l_{-1}\right)
$$

In this way, the wavelet subset encodes how much the data deviates from the model on which $\mathrm{P}$ was built. Then split $\lambda_{-1}$ into $\lambda_{-2}$ and $\gamma_{-2}$, and replace $\gamma_{-2}$ with the difference between $\gamma_{-2}$ and $P\left(\lambda_{-2}\right)$. After $\mathrm{n}$ steps, the original data is replaced by the wavelet representation $\left\{\lambda_{-n}, \gamma_{-n}, \cdots, \gamma_{-1}\right\}$. In order to solve the aliasing problem, the third step - update is introduced. Using an operator $U$ and the already computed wavelet set $\gamma_{-1}$ update $\lambda_{-1}$ so that a certain scalar quantity $\mathrm{Q}$ could be obtained,

$$
Q\left(g_{-1}\right)=Q\left(g_{-0}\right) \text {. }
$$

Therefore, we use the already computed wavelet set $\gamma_{-1}$ to update $\lambda_{-1}$ so that the latter preserves $\mathrm{Q}$. In this way, an operator $\mathrm{U}$ can be constructed and updating $\lambda_{-1}$ as

$$
l_{-1}:=l_{-1}+U\left(g_{-1}\right)
$$

The original data could be decomposed as

$$
l_{0}=\underbrace{\text { 恖 }}_{i} l_{i}+g_{i},(i=-n \sim-1)
$$

The above steps illustrate the basic idea of lifting. One property of lifting is that the inverse wavelet transform can immediately be found by undoing the operations of the forward transform. In practice, this comes down to simply reversing the order of the operations and changing each + to $\mathrm{a}-$ and vice versa. 


\subsubsection{Detection algorithm and its implementation}

This section would analyze the image to determine the mould core apple. The original RGB (Red, Green, Blue; the native space for color representation in computers) image was converted to grayscale image before processing. The $\mathrm{X}$-ray image could be transformed into a low frequency and three high frequency images by LWT. The low frequency image always represents the approximate character of the image, which can represent the original image with low noise, while the high frequency image always represents the noise and the points of discontinuity.

The procedure of the detection method is described as,

(1) Extract the low frequency image using LWT that represents the feature of the apple's image (Fig.1 (b), Fig.2 (b)).

(2) Enhance the image using histogram equalization in order to recognize the mould core (Fig.1 (c), Fig.2 (c)).

(3) Detect the apple's edge using Canny operator to get the apple core's diameter (Fig.1 (d), Fig.2 (d)), from which we can clearly obtain that the diameter of mould core apple is longer than the normal one's.

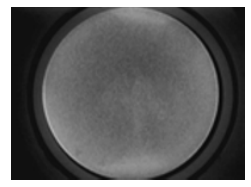

(a) original image

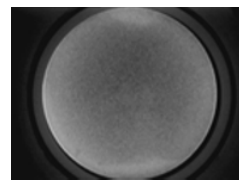

(a) original image

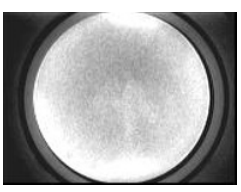

(b) low scale image

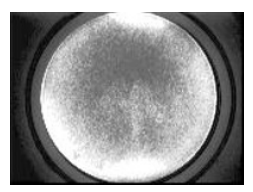

(c) enhanced image

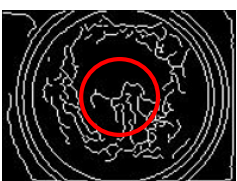

(d) edge detection image Fig. 1: X-ray images of a mould core apple

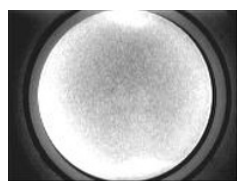

(b) low scale image

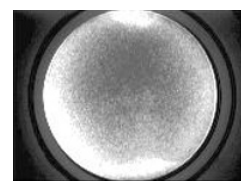

(c) enhanced image

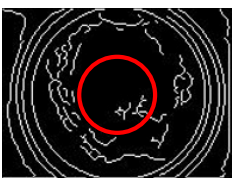

(d) edge detection image

Fig.2: X-ray images of a normal apple

(4) Calculate the diameter of the apple core by the statistical method counting the pixels corresponding to the mould core (the pixel value is one in the binary image) in four directions (Fig.3), the algorithm is showed as the equation (6). 


\begin{tabular}{|l|l|l|l|}
\hline 1 & 1 & 0 & 1 \\
\hline 1 & 1 & 1 & 1 \\
\hline 1 & 0 & $\rightarrow 1$ & 1 \\
\hline 1 & 1 & 1 & 1 \\
\hline
\end{tabular}

Fig.3: Four directions in the detection

$$
m=\begin{aligned}
& \text { 辛 } m+1, \text { pixel value }=1 \\
& \frac{1}{1} m, \quad \text { pixel value }=0
\end{aligned}
$$

(5) Compare the value of $m$ with the threshold that has been set to sort the apple, if $\mathrm{m}>$ threshold we can classify the apple to mould core apple, otherwise to good one.

\section{RESULTS AND DISCUSSION}

The results of this research are the development of a new online feature detection algorithm based on LWT, construction a detection system using machine vision and X-ray that can obtain the internal apple's image.

Table 1 shows the time consuming using different algorithms to detect the mould core. It illustrates that LWT is less-consuming than space domain method (contrast enhancement). We can clearly notice that the time consuming is less than 0.03 seconds by the frequency domain algorithm (LWT), which is quick enough to detect the quality in the on-line system.

Table 1. Time cost in different algorithms.

\begin{tabular}{ccccccc}
\hline \multirow{2}{*}{ Time cost } & \multirow{2}{*}{ Contrast enhancement } & \multicolumn{5}{c}{ LWT (Wave name) } \\
\cline { 3 - 7 } & & Haar & Db2 & Db4 & Lazy & 9.7 \\
\hline $1^{\text {st }}$ & 0.1424 & 0.0235 & 0.0245 & 0.0287 & 0.0225 & 0.0269 \\
$2^{\text {en }}$ & 0.1427 & 0.0229 & 0.0295 & 0.0276 & 0.0225 & 0.0265 \\
$3^{\text {rd }}$ & 0.1427 & 0.088 & 0.0238 & 0.0274 & 0.0224 & 0.0263 \\
Average & 0.1426 & 0.0251 & 0.0259 & 0.0279 & 0.0225 & 0.0266 \\
\hline
\end{tabular}

The unit of time cost is $s$.

Table 2 illustrates the detection results. The apple could be sorted at the accuracy of $92 \%$. Generally, the failed detection is aroused when the apple did not sharply face the $\mathrm{X}$-ray radiator.

Table 2. Detection results of the experiment.

\begin{tabular}{ccccc}
\hline Sample & Number & Detected & Undetected & Accuracy rate \\
\hline Normal & 72 & 69 & 3 & $92 \%$ \\
Mould core & 28 & 23 & 5 & \\
\hline
\end{tabular}


Overall, the results reveal that X-ray image is available as a nondestructive method used for detecting the apple's internal quality. Although this paper focused only on apple, the algorithm could also be used for pear, peach, pineapple and kiwi fruit. Moreover, the algorithm can be polished by other methods that need more study to promote its robust and accuracy.

\section{CONCLUSIONS AND FUTURE WORKS}

From the experimental results, the following conclusions can be drawn.

(1) The X-ray can obtain clear internal image of apple, which can be processed to detect the quality.

(2) Lifting wavelet transform is an efficient way to process the X-ray image, which can easily reduce the noise and enhance the image.

(3) The detection algorithm can quickly (less than 0.03 seconds per apple) and accurately detects the mould core apples, with an accuracy of $92 \%$ in the experiment.

The results of this research demonstrate the feasibility of an on-line X-ray based sensor for detecting internal quality of apple. In addition, the other works can be done at the automatic conveyor to push the internal quality detection to a new stage.

\section{ACKNOWLEDGEMENTS}

This research was supported by the Excellent Young Scientists Fund of Northwest A\&F University (01140303) and Postdoctoral Fund Project of China (20060401012). The authors would like to thank Mr. Fengtao Chen for his assistance in the preparation of the apple samples.

\section{REFERENCES}

Blasco J, Aleixos N, Gómez J, Moltó E. Citrus Sorting By Identification of The Most Common Defects Using Multispectral Computer Vision. Journal of Food Engineering, 2007, 83: 384-393 (in Chinese)

Daubechies I. Ten Lectures on Wavelets. SIAM, 1992

Han D, Tu R, Lu C, Liu X, Wen Z. Nondestructive Detection of Brown Core in the Chinese Pear 'Yali' by Transmission Visible-NIR Spectroscopy. Food Control, 2006, 17: 604-608

Muramatsu N, Sakurai N, Wada N, Yamamoto R, Takahara T, Ogata T, Tanaka K, Asakura T, Ishikawa-Takano Y, Nevins D J. Evaluation of Fruit Tissue Texture and Internal Disorders by Laser Doppler Detection. Postharvest Biology and Technology, 1999, 15: 83-88 
Slaughter D C, Obenland D M, Thompson J F, Arpaia M L, Margosan D A. Non-destructive Freeze Damage Detection in Oranges Using Machine Vision and Ultraviolet Fluorescence. Postharvest Biology and Technology, 2008, 48: 341-346

Sweldens W. Lifting Scheme: A New Philosophy in Biorthogonal Wavelet Constructions. Wavelet Applications in Signal and Image Processing III. 1995: 68-79

Sweldens W. The lifting scheme: A Custom-Design Construction of Biorthogonal Wavelets. Applied and Computational Harmonic Analysis, 1996, 3: 186-200

Thomas P, Kannan A, Degwekar V H, Ramamurthy MS. Non-destructive Detection of Seed Weevil-infested Mango Fruits By X-ray Imaging. Postharvest Biology and Technology, 1995, 5: 161-165

Thybo A K, Jespersen S N, Lærke E, Stødkilde-Jørgensen H J. Nondestructive Detection of Internal Bruise and Spraing Disease Symptoms in Potatoes Using Magnetic Resonance Imaging. Magnetic Resonance Imaging, 2004, 22: 1311-1317

Toyofuku N, Schatzki T F. Image Feature Based Detection of Agricultural Quarantine Materials in X-ray Images. Journal of Air Transport Management, 2007, 13: 348-354

Yang F Z, Wang H B, Yang Q, Wang Z. Wavelet Transform and Its Application in the Processing of Fruit Image. Transactions of The Chinese Society of Agricultural Machinery, 2005, 36: 61-64 (in Chinese)

Yang F Z, Wang Z, Yang Q, Zhang Y N. Application of Wavelet Transform-based Wiener Filtering Method to Denoise in Agricultural Product images. Transactions of the Chinese Society of Agricultural Engineering, 2007, 23: 145-150 (in Chinese)

Yi J H, Qiu N X, Hu B, Zhu Z B. Research of the Fumaric Acid in Concentrated Apple Fuice. China Fruit and Vegetable, 2001: 24-24 (in Chinese)

Ying Y B, Liu Y D, Fu X P. Sugar Content Prediction of Apple Using Near-Infrared Spectroscopy Treated by Wavelet Transform. Spectroscopy and Spectral Analysis, 2006, 26: 63-66 (in Chinese)

Zerbini P E, Grassi M, Cubeddu R, Pifferi A, Torricelli A. Nondestructive Detection of Brown Heart in Pears by Time-resolved Reflectance Spectroscopy. Postharvest Biology and Technology, 2002, 25: 87-97

Zhang C H, Liu C Q, Liu M H, Wang Q. A Study on Inspecting Internal Quality of Rambuta Using X-ray CT Imaging. Acta Agriculturae Universitis Jiangxiensis, 2005,27,06 : 939-942 (in Chinese)

Zhang J, Yuan X L, Zhao L, Cheng S L. The Rule and Control of Mould Core of Apple. Northwest horticulture: fruit trees, 2008, 1: $52-52$ (in Chinese) 